(C) Masson, Paris, 1979.

\title{
Sur les Muspiceidae (Nematoda-Dorylaimina)
}

\author{
par O. BAIN et A.-G. CHABAUD \\ Laboratoire de Zoologie (Vers) associé au C.N.R.S., Muséum national d'Histoire naturelle, \\ 43, rue Cuvier, F 75231 Paris Cedex 05
}

\section{Résumée.}

Les Muspiceidae, parasites de la membrane alaire des chauves-souris, n'étaient connus que par un spécimen immature (genre Riouxgolvania) et par la reconstitution de coupes histologiques sériées (genre Lukonema).

Nous disposons maintenant d'un matériel plus abondant et plus varié, et nous avons vu des spécimens vivants.

Il a été possible de confirmer ou d'infirmer les interprétations morphologiques fournies précédemment et d'aboutir aux conclusions suivantes.

Les Muspiceidae ont tous :

— un œsophage court et filiforme portant à son extrémité postérieure des glandes qui paraissent homologues des "primary glands" des larves préparasitaires de Mermithoïdes (Chitwood et Chitwood, p. 95).

Les 2 glandes ventro-latérales sont variables (dédoublées, simples, fusionnées, atrophiées), alors que la glande dorsale, très constante, est transformée en un sinus osmo-excréteur.

- un intestin transformé en trophosome;

- une large poche utérine dans laquelle s'effectue tout le début du développement larvaire ;

- une expulsion de larves mûres hors de l'hôte par un petit cratère cutané creusé par l'extrémité antérieure du Ver.

Les Muspiceidae sont bien des Dorylaimina au sens de Chitwood et ne paraissent pas très éloignés des Mermithoidea.

Mermithoidea, Muspiceoidea (Muspiceidea et Robertdollfusidae) (1) et Trichuroidea paraissent ainsi un groupe naturel de formes parasites issues de Dorylaimoidea.

Les Muspiceides comprennent actuellement 4 genres :

1. - Muspicea Sambon, parasite intra-tissulaire de la Souris domestique.

Glandes primaires œsophagiennes ventrales doubles. Phasmides petites. Hermaphrodisme protérandrique. Vulve atrophiée. Larves émises par rupture de l'extrémité antérieure du Ver.

(1) Les Phlyctainophoridae placés à titre hypothétique dans la même superfamille sont en réalité des Philometridae (Mudry et Dalley, 1969).

Accepté le 5 février 1979. 
Bouche de la larve inerme. Infestation de l'hôte vraisemblablement par voie orale. Espèce type: $M$. borreli Sambon, 1925.

2. - Riouxgolvania Bain et Chabaud, parasite de la membrane alaire des chauves-souris en Europe et en Australie.

Glandes primaires œsophagiennes ventrales absentes. Phasmides petites. Hermaphrodisme protérandrique. Vulve fonctionnelle. Larves atteignant l'extrémité antérieure du Ver par migration intracuticulaire. Bouche de la larve armée. Infestation de l'hôte vraisemblablement par voie cutanée.

Espèce type: Riouxgolvania rhinolophi Bain et Chabaud, 1968.

Autres espèces: $R$. nyctali n. sp., $R$. beveridgei $\mathrm{n}$. sp.

3. - Lukonema Chabaud et Bain, parasite de la membrane alaire des chauves-souris en Amérique du Sud.

Glandes primaires œsophagiennes ventrales fusionnées en un seul cordon. Phasmides avec organes (?) d'absorption internes. Hermaphrodisme protérandrique. Vulve fonctionnelle. Larves atteignant l'extrémité antérieure du Ver par migration intracuticulaire. Bouche de la larve armée. Infestation de l'hôte vraisemblablement par voie cutanée.

Espèce type: Lukonema lukoschusi Chabaud et Bain, 1974.

4. - Pennisia n. gen., parasite de la membrane alaire des chauves-souris au Kenya.

Glandes primaires œsophagiennes ventrales simples. Phasmides avec organes (?) d'absorption externes. Sexes séparés. Vulve présente. Larves inconnues.

Espèce type: Pennisia nagorseni n. sp.

\section{Summary.}

\section{On the Muspiceidae (Nematoda-Dorylaimina).}

The Muspiceidae which parasitize the skin wing of bats were previously known only from examinations of one immature specimen (Riouxgolvania) and histological serial sections (Lukonema).

In this paper additional adult and larval specimens of Riouxgolvania rhinolophi, two new species ( $R$. nyctali, $R$. beveridgei) and a new genus (Pennisia nagorseni) are described. Museum specimens of Muspicea borreli Sambon, 1925 were also examined and differences in interpretation from those of Brumpt in 1930 are discussed.

Common features of theses parasites were :

- a short, thread-like oesophagus with glands at the posterior end homologous to the "primary glands" of the preparasitic larvae of the Mermithidae (see Chitwood p. 95). The two ventro-lateral glands were variable in shape (twin, simple, coalescent or atrophic).

A constant feature of the dorsal gland was an osmo-excretory sinus.

- a gut transformed into a trophosoma;

- initial larval development in a large uterine pouch:

- escape of mature larvae from the host through a small cutaneous ulcer induced by the anterior end of the worm.

Arguments are presented that Muspiceidae are actually Dorylaimina sensu Chitwood, and are close to Mermithoidea. It is concluded that Mermithoidea, Muspiceoidea (Muspiceidae and Roberdollfusidae) (2) and Trichuroidea are a natural group of invertebrate or vertebrate parasites.

(2) Phlyctainophoridae hypothetically classified in the same superfamily are actually Philometridae (Mudry and Dalley, 1969). 
It is proposed that Muspiceidae now includes four genera:

1. - Muspicea Sambon, parasites of tissues of the domestic mouse.

Twin ventral primary oesophageal glands. Phasmids small. Proterandric hermaphrodism. Atrophic vulva. Larvae expelled from the host by the splitting of the anterior end of the female. Smooth larval mouth. Infection of the host probably per os.

Type species: $M$. borreli Sambon, 1925.

2. - Rivuxgolvania Bain and Chabaud, parasites of the skin wing of bats in Europe and Australia.

Ventral primary oesophageal glands absent. Phasmids small. Proterandric hermaphrodism. Functional vulva. Larvae reaching the anterior end of the worm by intercuticular migration. Larvae with buccal armature. Infection of the host probably through the skin. Type species: Riouxgolvania rhinolophi Bain and Chabaud, 1968.

Other species: $R$. nyctali $\mathrm{n}$. sp., $R$. beveridgei $\mathrm{n}$. sp.

3. - Lukonema Chabaud and Bain, parasites of the skin wing of bats in South America.

Ventral primary oesophageal glands fusing on a line. Phasmids with presumably absorbing internal organs. Proterandric hermaphrodism. Functional vulva. Larvae reaching the anterior end of the worm by an intercuticular migration. Larvae with buccal armature. Infection of the host probably through the skin.

Type species: Lukonema lukoschusi Chabaud and Bain, 1974.

4. - Pennisia n. gen., parasites of the skin wing of bats in Kenya.

Simple ventral primary oesophageal glands. Phasmids with presumably absorbing external organs. Diecious. Vulva present. Larvae unknown.

Type species: Pennisia nagorseni n. sp.

Nous devons à la générosité de nos collègues, David Nagorsen et Roy C. Anderson, la communication d'intéressants Nématodes, parasites de la membrane alaire de chauvessouris africaines. Il s'agit d'un nouveau genre de Muspiceidae, que nous nommerons Pennisia n. gen. en raison d'étranges formations d'aspect plumeux insérées dans les phasmides.

Il est apparu que les quatre genres constituant actuellement la famille des Muspiceidae, bien qu'ils aient chacun des éléments très originaux, ont une réelle homogénéité et que l'interprétation de leur anatomie est facilitée par la comparaison entre les structures des différents genres.

Avant de décrire le nouveau genre, nous avons donc:

1. - revu le matériel de Muspicea récolté par Brumpt ;

2. - effectué de nouvelles récoltes de Riouxgolvania chez différents Microchiroptères (France et Australie) et complété l'étude de ce genre, qui n'était connu que par une femelle immature ;

3. - repris notre matériel Lukonema pour confirmer ou modifier l'interprétation des structures observées.

Le point le plus inattendu qui résulte de ces comparaisons est que la cellule excrétrice correspond dans les quatre genres à la première glande œsophagienne dorsale transformée en un appareil d'osmorégulation se déversant dans la lumière œsophagienne. 


\section{Études morphologiques}

\section{Genre Muspicea.}

Le matériel observé, $M$. borreli Sambon, 1925, est celui étudié par Brumpt en 1930 et conservé au M.N.H.N. de Paris.

1. - La figure 1, A à $\mathrm{D}$, représente la région antérieure d'une femelle (fig. 1, $A=$ fig. 5 de Brumpt).

Le « cordon cellulaire dorsal» (1.) est, dans notre interprétation, comme dans celle de Brumpt, un intestin modifié en trophosome, appendu au tégument aux deux extrémités du corps.

Sur d'autres points, notre interprétation diffère de celle de Brumpt :

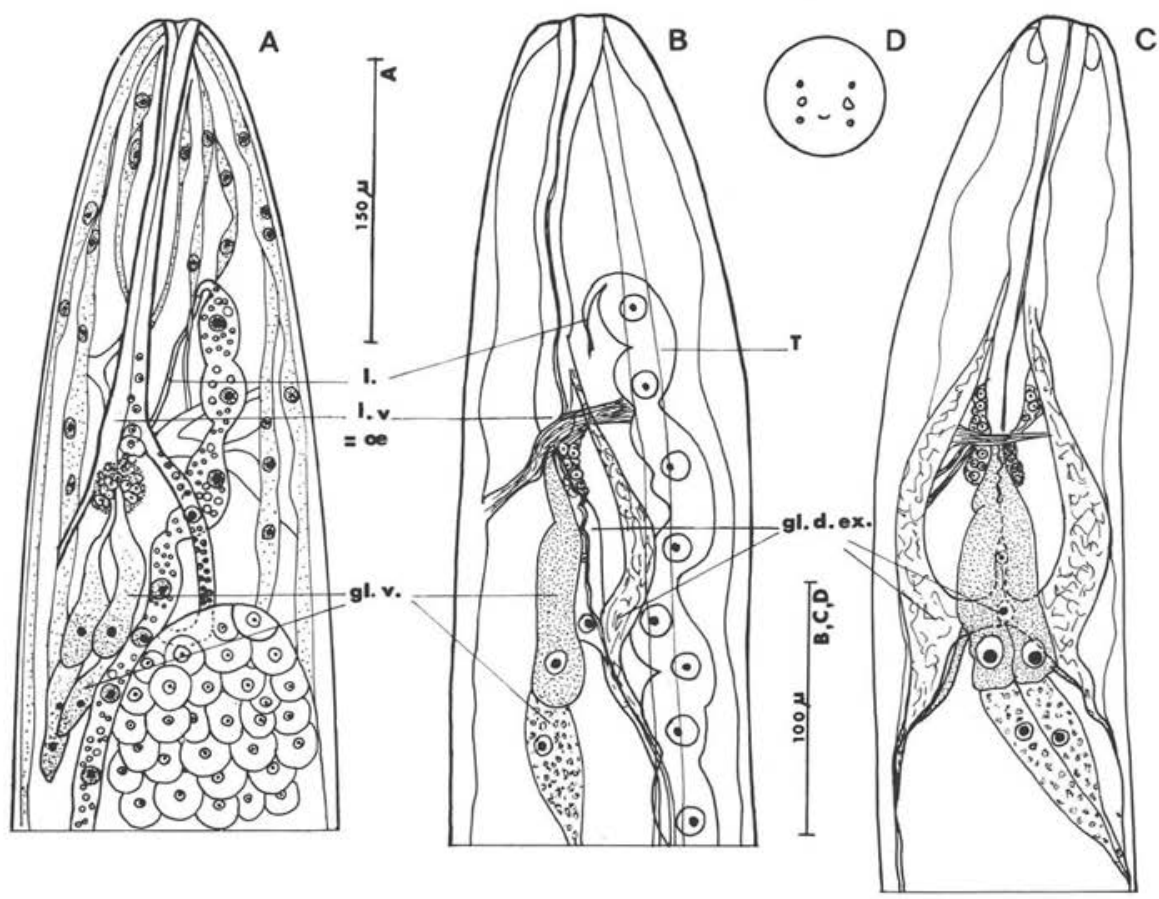

Fig. 1. Muspicea borreli, région antérieure d'une ?. A : copie de la fig. 5 de Brumpt, 1930 ; $\mathrm{B}$ et $\mathrm{C}$ : vues latérale et ventrale, montrant les 5 glandes osophagiennes, dont la dorsale est traversée par un conduit excréteur; D : tête, vue apicale.

(A : éch. $150 \mu \mathrm{m} ; \mathrm{B}, \mathrm{C}$ et D : éch. $100 \mu \mathrm{m}$ )

Symboles : $1=$ ligament antérieur du trophosome $; \propto=œ$ sophage $(=$ ligne médiane ventrale, 1.v. selon Brumpt); gl.v. = cellules glandulaires œsophagiennes ventrales; gl.d.ex. $=$ cellule glandulaire œsophagienne dorsale, osmoexcrétrice; $\mathrm{T}=$ trophosome. 
a) L'œsophage est présent ; il a été confondu précédemment avec les "lignes médianes ventrale et dorsale "; cet œsophage est court $(168 \mu \mathrm{m})$, fibreux, avec une mince lumière et se termine au niveau de l'anneau nerveux; la bouche est déportée ventralement (3).

b) Les "glandes ventrales" (Gl. v.) sont appendues à la fin de l'œsophage et non à la «ligne médiane ventrale "; elles forment deux chaînes latéro-ventrales de deux cellules chacune et nous les assimilons à 4 glandes œsophagiennes latéro-ventrales.

c) L'appareil excréteur existe: il est formé par une volumineuse cellule, appliquée contre la face dorsale des glandes œsophagiennes latéro-ventrales antérieures et appendue comme elles à l'œsophage; nous interprétons cette cellule comme une glande œsophagienne dorsale profondément modifiée. Cette cellule forme antérieurement et postérieurement deux paires de cornes latérales d'aspect vacuolaire; les deux cornes antérieures vont rejoindre le tissu fibreux œsophagien, un peu en avant de l'anneau nerveux; les deux cornes postérieures fusionnent avec les cordes latérales; la cellule dorsale est traversée par un canal longitudinal qui, en avant, débouche dans la lumière œsophagienne, et qui bifurque en arrière dans les cornes postérieures pour constituer dans les deux champs latéraux les canaux excréteurs longitudinaux.

2. - La larve mûre (fig. $4, A$ à $D$ ) montre des structures comparables à celles de l'adulte : bouche ventrale, canal œsophagien auquel est appendue postérieurement une volumineuse cellule, la glande excrétrice dorsale, d'où partent deux branches latérales épaisses ; ventralement à cette cellule, un petit massif cellulaire (? quatre cellules) pourrait correspondre aux deux glandes œsophagiennes latéro-ventrales. L'intestin est déjà transformé en trophosome (fin cordon cellulaire dorsal sans connexion avec l'œsophage). A 28-30 $\mu \mathrm{m}$ de l'extrémité postérieure du corps se trouvent deux gros noyaux latéraux que nous interprétons comme des phasmides et qui semblent correspondre aux deux volumineuses cellules vacuolaires que Brumpt observe chez la femelle à $350 \mu \mathrm{m}$ de l'extrémité caudale. Chez la larve, les pores phasmidiens sont visibles à 15-20 $\mu \mathrm{m}$ des pointes caudales. L'ébauche génitale, dont deux noyaux sont plus volumineux, est située dans la partie moyenne du corps.

Une des larves mûres est longue de $330 \mu \mathrm{m}$ et large de $17 \mu \mathrm{m}$; elle est entourée par une exuvie ; l'anneau nerveux, le noyau de la glande excrétrice dorsale et l'ébauche génitale sont à $78 \mu \mathrm{m}, 14 \bar{\jmath} \mu \mathrm{m}$ et $200 \mu \mathrm{m}$ de l'apex. La queue est bifide et chaque pointe latérale est incisée à l'apex.

\section{Genre Riouxgolvania.}

Le matériel étudié est hétérogène et comprend trois espèces.

A. - Riouxgolvania rhinolophi Bain et Chabaud, 1968.

Cette espèce a été retrouvée à diverses reprises :

(3) L'orientation du ver est basée sur l'anneau nerveux, qui est incliné vers la face ventrale. 
- de nombreuses femelles, immatures ou mûres, récoltées chez Miniopterus schreibersi, à Moulis (Ariège), en mai 1978, lot 90 à $107 \mathrm{EN}$; ces femelles forment des kystes situés dans la membrane alaire, près des flancs ;

- une femelle gravide, également parasite de $M$. schreibersi, récoltée en Corse par Léger et coll. (grotte de Sisco) en avril 1977, lot 270 JE;

- une femelle en deux morceaux, qui paraît n'avoir que quelques œufs, récoltée par C. Combes chez M. schreibersi, le 25 novembre 1966, à la grotte de Fuilla, à $480 \mathrm{~m}$ d'altitude, près de Prades (Pyrénées-Orientales), lot 37 NN.

L'holotype de $R$. rhinolophi provient de Rhinolophus euryale; nous croyons pouvoir assimiler notre nouveau matériel à cette espèce ; le mucron caudal, en particulier, qui est assez caractéristique, est identique.

R. rhinolophi n'était connue que par une femelle immature. Nous pouvons maintenant décrire la femelle mûre et les larves, et modifier l'interprétation de certaines structures.

a) Morphologie de la femelle (fig. 2).

L'anatomie de la femelle, telle qu'elle avait été interprétée, doit être modifiée ou complétée sur quatre points :

1. - L'œsophage : ce que nous avions décrit comme un œsophage, long de $700 \mathrm{um}$, est en réalité formé de deux organes - l'œsophage proprement dit, qui se termine en cul-de-sac juste en arrière de l'anneau nerveux et qui est long de 280 à $320 \mu \mathrm{m}$, un organe postœsophagien allongé, qui lui fait suite.

2. - L'appareil osmo-excréteur : les deux canaux chitinoïdes latéraux, visibles le long du corps, ne sont pas, comme nous en avions émis l'hypothèse, issus de deux glandes caudales modifiées; ils prennent naissance dans l'organe postœsophagien et se prolongent en avant par deux courtes branches.

L'organe postosophagien est lui-même bifurqué à ses deux extrémités; il est constitué essentiellement de deux canaux unis sur la ligne médiane, entourés de quelques rares noyaux de petite taille (? cellules nerveuses et de soutien) et par un très volumineux noyau postérieur et dorsal qui est vraisemblablement le noyau osmo. excréteur.

Cet organe, avec ses canaux, présente donc une structure tout à fait comparable à ce qui s'observe chez Muspicea et nous semble pouvoir être interprété aussi comme une glande œsophagienne dorsale modifiée en appareil excréteur.

Toutefois, Riouxgolvania présente deux particularités: existence d'une cellule à cytoplasme grenu appendue à la fin de la cellule osmo-excrétrice et qui pourrait correspondre à une deuxième glande dorsale; absence des glandes œsophagiennes latéro-ventrales, bien développées au contraire chez Muspicea.

3. - Le trophosome : sa structure et sa position sont identiques à celles de Muspicea et il correspond, croyons-nous, non au stichosome, mais à l'intestin. 


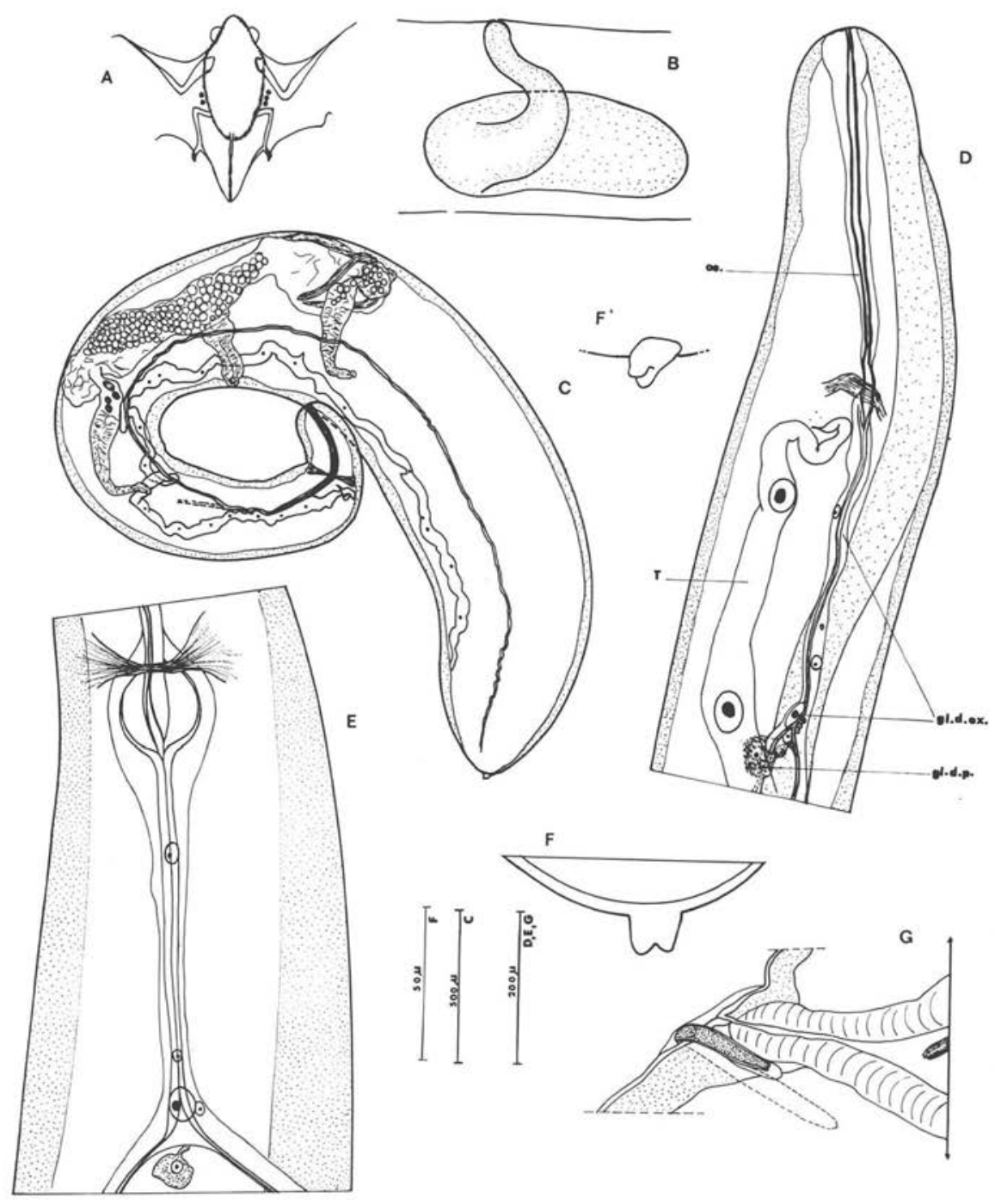

Fig. 2. Riouxgolvania rhinolophi. A : situation des kystes chez Miniopterus schreibersi ; B : position du ver mûr dans la membrane alaire (la tête perfore la face externe de l'aile); C : $ᄋ$, avec œufs et larves, vue latérale; D : région antérieure, vue latérale; E : fin de l'œsophage et glande œsophagienne dorsale osmo-excrétrice, vue médiane; $F$ et $F^{\prime}$ : mucron caudal, vue latérale et vue sublatérale; G : 1 larve sortie par la vulve et maintenue sous le feuillet cuticulaire externe.

(A et B : à main levée; C : éch. $100 \mu \mathrm{m} ; \mathrm{D}, \mathrm{E}, \mathrm{G}$ : éch $200 \mu \mathrm{m} ; \mathrm{F}$ : éch. $50 \mu \mathrm{m}$ ). Symboles : cf. fig. 1; gl.d.p. : cellule glandulaire œsophagienne dorsale postérieure. 
4. - L'appareil génital : les femelles mûres diffèrent des femelles immatures par le diamètre plus grand des $2 / 3$ postérieurs du corps, gonflés par l'utérus rempli de larves I, II et III.

Bien que l'ovéjecteur ait une structure peu différenciée, les larves mûres quittent l'utérus par cet organe et non par rupture du corps de la femelle, comme nous l'avions supposé. Mais la vulve, comme chez Lukonema, est recouverte par une couche cuticulaire et les larves s'insinuent entre cette couche et le feuillet cuticulaire du corps de la femelle ; elles migrent ainsi vers la tête, qui est en contact avec l'extérieur, au centre d'un petit cratère perforé dans la membrane alaire ; les larves mûres s'échappent à la hauteur de la bouche (fig. 3) et perdent alors, en général, leurs exuvies.

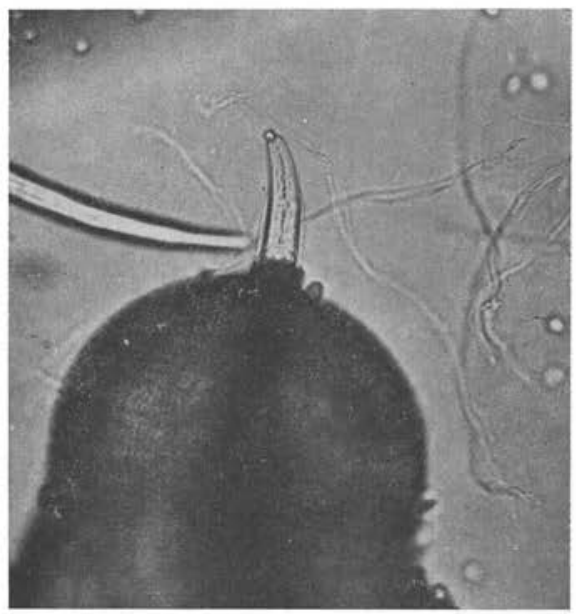

Fig. 3. Une larve infestante émergeant de la tête de Riouxgolvania rhinolophi et nombreuses exuvies larvaires autour' de la tête (préparation d'un ver vivant dans l'eau physiologique).

b) Morphologie larvaire (fig. 4, $E$ à $M$ ).

Trois stades larvaires s'observent dans l'utérus :

1. - Le stade I est long de $255 \mu \mathrm{m}$ et large de $18 \mu \mathrm{m}$; l'extrémité caudale est arrondie. L'anatomie interne est peu nette, et seuls sont visibles le noyau de la glande osmoexcrétrice, le trophosome intestinal et l'ébauche génitale subéquatoriale.

2. - Le stade II est long de 350 à $375 \mu \mathrm{m}$ sur $20 \mu \mathrm{m}$ de large ; il est enfermé dans la mue I ; la queue est bifide ; l'anatomie, identique à celle du stade III, est décrite au paragraphe suivant.

3. - Le stade III a les mêmes dimensions que le stade II ; il a des ailes latérales, bien visibles lorsqu'il a perdu ses exuvies; la queue se termine par 2 pointes et chaque pointe est bifide; la tête porte une armature saillante, formée par une petite arête allongée dans le plan médian, soutenue à ses deux extrémités par deux axes cuticulaires, dont la base est trifurquée; la bouche est déportée ventralement ; l'œsophage 


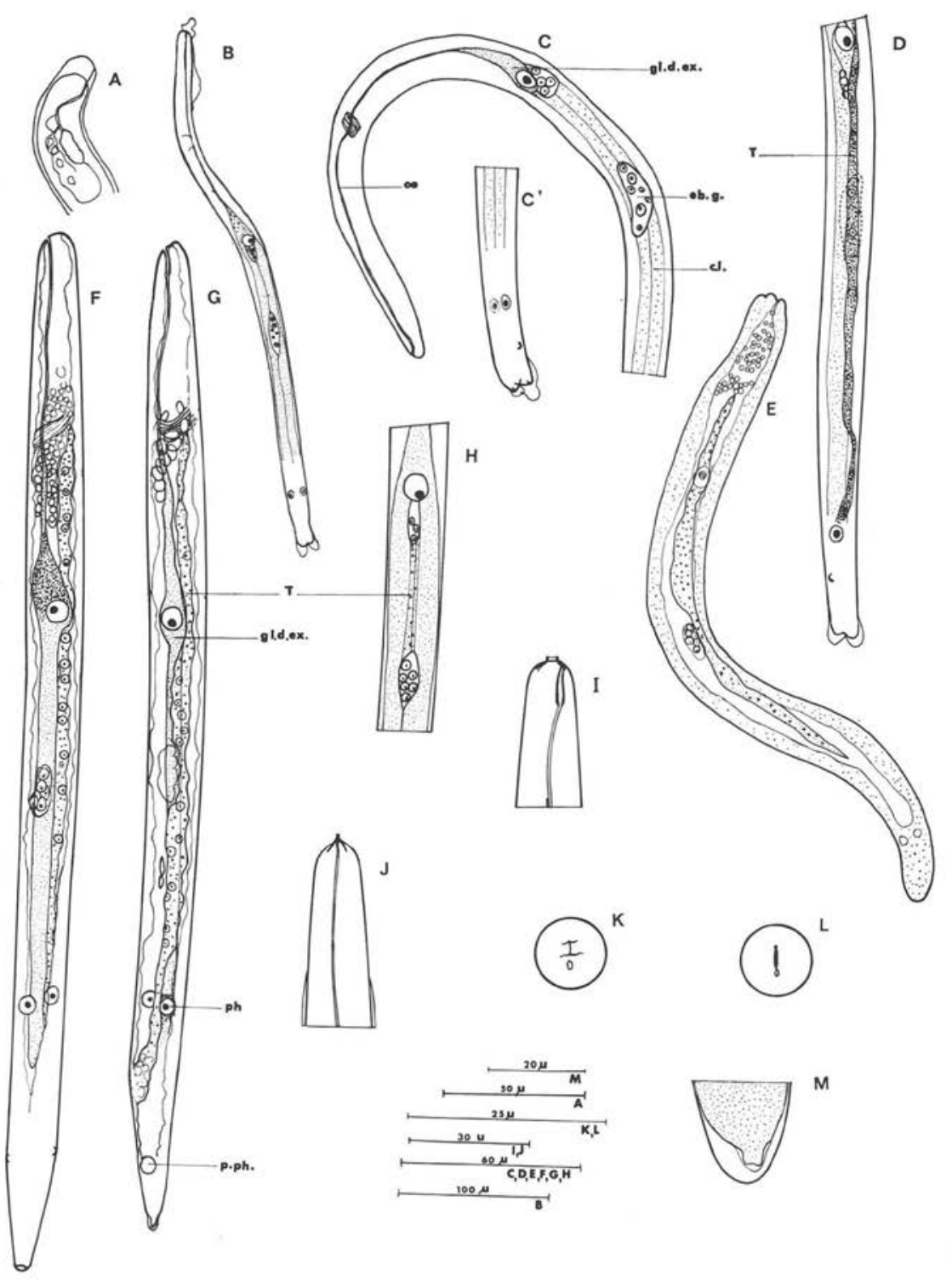

Fig. 4. A à D, larve infestante de Muspicea borreli; $\mathrm{A}$ : une extrémité antérieure, extraite de la figure 7 de Brumpt; B : aspect général, vue ventrale ; $\mathrm{C}$ et $\mathrm{C}^{\prime}$ : organisation interne, vue latérale; D : moitié postérieure du corps d'une autre larve montrant le trophosome. $\mathrm{E}$ à $\mathrm{M}$ : larves de Riouxgolvania rhinolophi; E : stade 1; F : stade 2 dans la mue 1; G : larve infestante dans les mues 1 et $2 ; \mathrm{H}$ : idem, région de la glande œsophagienne dorsale et de l'ébauche génitale, vue ventrale; I et $\mathrm{J}$ : stade 3 libéré des exuvies, région céphalique en vue latérale et médiane; $\mathrm{K}$ et $\mathrm{L}$ : vue apicale de la tête, superficielle et plus profonde; $\mathrm{M}$ : queue du stade 3 et exuvies 1 et 2 , en vue latérale.

(A : éch. $50 \mu \mathrm{m}$; B : éch. $100 \mu \mathrm{m}$; C, D, E, F, G, H : éch. $60 \mu \mathrm{m}$; I et J : éch. $30 \mu \mathrm{m}$; $\mathrm{K}$ et $\mathrm{L}$ : éch. $25 \mu \mathrm{m} ; \mathrm{M}$ : éch. $20 \mu \mathrm{m})$.

Symboles : cf. fig. 1 ; éb.g. = ébauche génitale ; c.l. = corde latérale; $\mathrm{ph}=$ phasmide ; p. ph. $=$ pore phasmidien. 
est prolongé en arrière de l'anneau nerveux par la volumineuse glande dorsale osmoexcrétrice, qui envoie deux branches latérales le long du corps; un massif de troisquatre cellules, appendu à la glande dorsale, pourrait être l'ébauche des deux glandes œsophagiennes latéro-ventrales, atrophiées chez l'adulte; le cordon intestinal dorsal est attaché antérieurement près de l'anneau nerveux et forme postérieurement un rectum atrophié ; l'ébauche génitale est à mi-corps ; les phasmides sont volumineuses. Chez un stade infestant long de $375 \mu \mathrm{m}$, les dimensions sont les suivantes : œsophage long de $68 \mu \mathrm{m}$, noyau de la glande œsophagienne dorsale à $140 \mu \mathrm{m}$ de la tête, rectum à $48 \mu \mathrm{m}$ de l'extrémité caudale, noyaux et pores phasmidiens à 90 et $25 \mu \mathrm{m}$ de l'extrémité caudale, ébauche génitale à $180 \mu \mathrm{m}$ de la tête.

B. - Riouxgolvania nyctali n. sp. (fig. 5, $A$ à $C$ ).

Le matériel étudié a une double origine. Le lot 467 RL a été récolté par le $\mathrm{D}^{\mathrm{r}}$ Lukoschus chez Nyctalus noctula en Hollande, le 30 août 1973, et comprend une femelle en bon état (holotype) et une femelle en deux morceaux, toutes deux avec des œufs en segmentation avancée et une dizaine de larves. Le lot 91 EN a été récolté à Moulis (Ariège) chez Myotis oxygnathus (4) et comprend une femelle mûre située dans un kyste à l'oreille.

a) Morphologie de la femelle.

Femelle holotype longue de $1880 \mu \mathrm{m}$ et large de $250 \mu \mathrm{m}$; anneau nerveux à $200 \mu \mathrm{m}$ de l'apex ; œsophage long de $200 \mu \mathrm{m}$, auquel est appendue la glande osmoexcrétrice, bien délimitée et subsphérique, longue de $60 \mu \mathrm{m}$; vulve à $650 \mu \mathrm{m}$ de la tête et vagin non musculeux, long de $100 \mu \mathrm{m}$; trophosome intestinal débutant à la hauteur de l'anneau nerveux et finissant à $140 \mu \mathrm{m}$ de l'extrémité caudale ; celle-ci est formée par une pointe tronquée, qui porte quatre reliefs apicaux peu saillants.

b) Morphologie larvaire.

Deux larves en mue situées dans l'utérus sont longues de 220 et $230 \mu \mathrm{m}$ et larges de $18 \mu \mathrm{m}$; le stichocyte osmo-excréteur est à $85 \mu \mathrm{m}$ et $92 \mu \mathrm{m}$ de la tête ; la queue est bifide.

Ces spécimens diffèrent de $R$. rhinolophi par les caractères indiqués p. 219. Ils constituent donc une espèce nouvelle, Riouxgolvania nyctali n. sp.

C. - Riouxgolvania beveridgei n. sp. (fig. 5, D à K).

4 immatures et 3 mûres (dont l'holotype) ont été récoltées chez un Miniopterus schreibersi ô en Australie, dans le Nord Queensland, à $120 \mathrm{~km}$ au nord de Townsville (Paluma Dam), en novembre 1978: kystes situés dans l'épaisseur du patagium au niveau des tibias et des pieds. Holotype déposé au South Australian Museum (Adélaïde).

(4) Le même Myotis avait d'autres kystes, également situés sur les oreilles, et contenant d'autres Riouxgolvania sp.; ceux-ci, distincts de $R$. nyctali n. sp. par la taille plus grande et l'extrémité caudale différente (4 tubercules), sont trop mûrs et lysés par les réactions de l'hôte pour pouvoir être étudiés. 


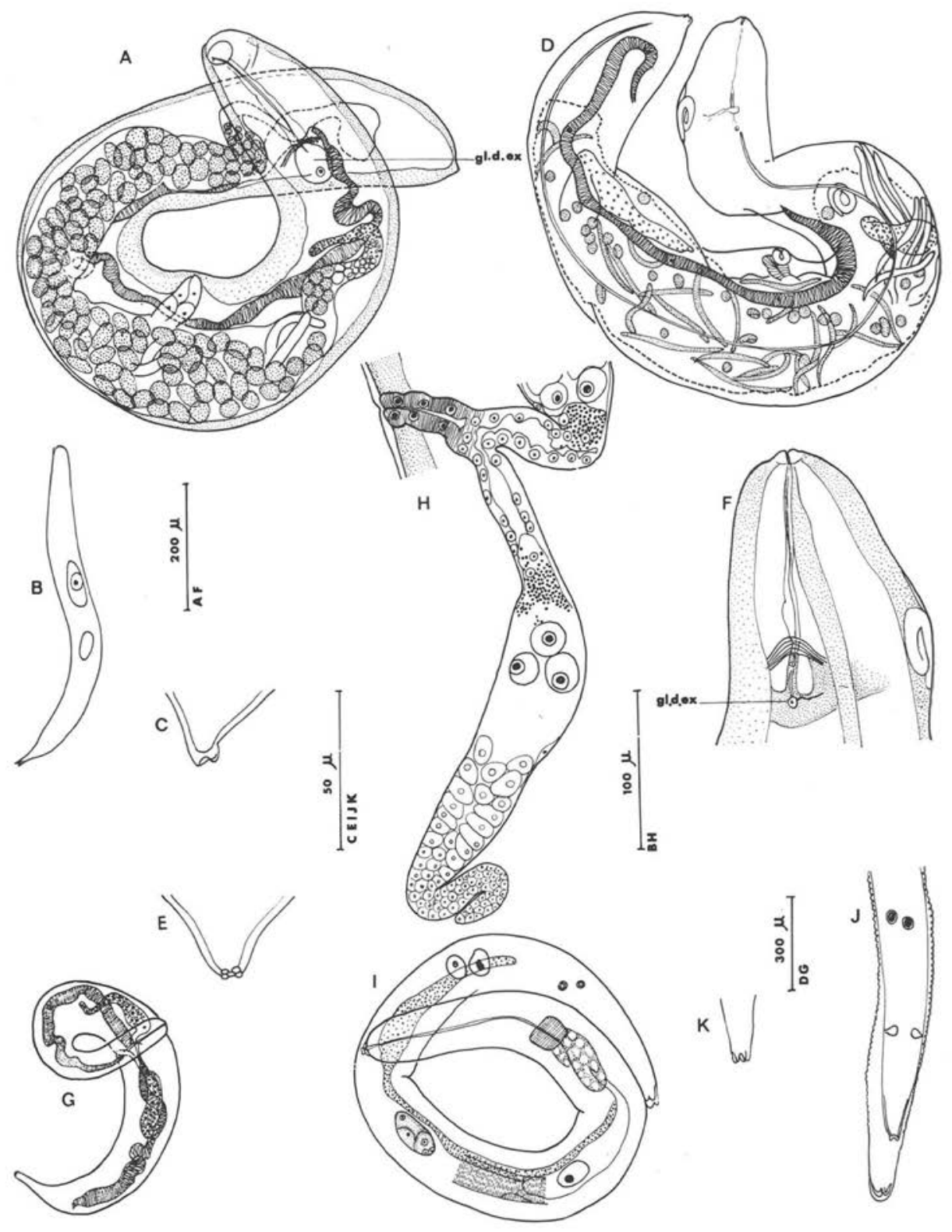

Fig. 5. A à C, Riouxgolvania nyctali n. sp. A : 1 , vue latérale ; B : 1 larve mûre intrautérine ; C : extrémité caudale d'une $q$, vue latérale. D. à $\mathrm{K}, R$. beveridgei $\mathrm{n} . \mathrm{sp}$.; $\mathrm{D}$ : $q$ holotype, vue latérale (les larves ponctuées sont dans l'utérus; les autres circulent entre les 2 assises cuticulaires) ; E : idem, extrémité caudale, vue latérale; F : idem, région œsophagienne et glande œsophagienne dorsale osmo-excrétrice; G : jeune ; ; H : idem, appareil génital ; I : larve mûre, vue latérale; J : idem, région caudale entourée de 2 exuvies, vue médiane; $\mathrm{K}$ : idem, extrémité caudale, vue submédiane.

(A et F : éch. $200 \mu \mathrm{m}$; B et H : éch. $100 \mu \mathrm{m}$; C, E, I, J, K : éch. $50 \mu \mathrm{m}$; D et G : éch. $300 \mu \mathrm{m}$ ) Annales de Parasitologie humaine et comparée (Paris), t. 54, $\mathrm{n}^{\circ} 2$ 
a) Morphologie de la femelle.

Habitus comparablé à celui de l'espèce type ; chez tous les spécimens, l'extrémité postérieure du corps s'amincit et forme un petit dôme orné par 4 tubercules saillants et arrondis, 2 latéro-ventraux et 2 latéro-dorsaux.

Holotype long de $3 \mathrm{~mm}$ et large de $420 \mu \mathrm{m}$, anneau nerveux à $300 \mu \mathrm{m}$ de l'extrémité antérieure, œsophage long de $325 \mu \mathrm{m}$ et noyau de la glande œsophagienne dorsale à $70 \mu \mathrm{m}$ de la fin de l'œsophage ; trophosome (abîmé dans la région antérieure) se terminant à $300 \mu \mathrm{m}$ de l'extrémité caudale ; vulve à $1450 \mu \mathrm{m}$ de la tête et vagin long de $100 \mu \mathrm{m}$.

Les autres $q$ mûres sont longues de $3 \mathrm{~mm}$ et $2,25 \mathrm{~mm}$, et larges respectivement de $300 \mu \mathrm{m}$ et $320 \mu \mathrm{m}$; la glande œsophagienne dorsale est longue de $75 \mu \mathrm{m}$; le trophosome est attaché au tégument du corps à la hauteur de l'anneau nerveux.

La plus petite femelle immature est longue de $1,58 \mathrm{~mm}$ et large de $170 \mu \mathrm{m}$; l'appareil génital, peu développé, n'occupe que la région moyenne du ver (fig. 5, $G$ et $H$ ) ; les 2 tubes utérins sont très courts et à paroi épaisse ; ils sont fermés vers les ovaires par 2 massifs bien délimités, contenant de très nombreux spermatozoïdes punctiformes et, peut-être, quelques spermatides; ces spermatozoïdes ne peuvent pas provenir de la fécondation par un autre ver, car les tubes utérins et le vagin ne communiquent pas et sont simplement accolés.

\section{b) Morphologie larvaire (fig. 5, I à K).}

Une larve mûre, en migration entre les 2 couches cuticulaires de la $q$ holotype, est longue de $310 \mu \mathrm{m}$ ef large de $22 \mu \mathrm{m}$. La bouche est subventrale ; la tête est armée par 2 formations cuticulaires médianes, dont la base est trifurquée. Anneau nerveux, noyau de la glande œsophagienne et appareil génital à $60 \mu \mathrm{m}, 118 \mu \mathrm{m}$ et $170 \mu \mathrm{m}$ de l'apex, noyaux et pores des phasmides à $75 \mu \mathrm{m}$ et $45 \mu \mathrm{m}$ de l'extrémité caudale. Queue à 4 tubercules, 2 latéro-ventraux et 2 latéro-dorsaux.

Des larves extraites d'une autre $q$ mûre montrent la présence de 2 exuvies; l'extrémité postérieure de l'exuvie 1 est arrondie, celle de l'exuvie 2 est divisée en 4 languettes égales.

Ces spécimens diffèrent de $R$. rhinolophi et de $R$. nyctali n. sp. par les caractères indiqués p. 219 ; ils constituent une nouvelle espèce, $R$. beveridgei n. sp. dédiée à notre collègue australien I. Beveridge. 


\section{Caractères distinctifs des 3 espèces de Riouxgolvania}

- R. rhinolophi : Glande dorsale étroite et aussi longue que l'œsophage (250-330 $\mu \mathrm{m})$; extrémité caudale constituée par un mucron cylindrique long de $20 \mu \mathrm{m}$, incisé à l'apex.

Larve infestante longue de $350-375 \mu \mathrm{m}$, à queue bifide, chaque pointe étant incisée à l'apex.

Hôtes: Rhinolophus euryale (hôte type), grotte de Pouade (Pyrénées-Orientales) ; Miniopterus schreibersi, diverses grottes (Ariège, Corse, Pyrénées Or.).

- R. nyctali n. sp.: Glande dorsale sphérique et courte $(60 \mu \mathrm{m})$; pointe caudale de $5 \mu \mathrm{m}$ de long, tronquée, avec 4 reliefs terminaux peu saillants.

Larve infestante petite $(220 \mu \mathrm{m})$, à queue bifide.

Hôtes: Nyctalus noctula (hôte type), Hollande; Myotis oxygnathus, à Moulis.

- $R$. beveridgei n. sp. : Glande dorsale courte $(75-80 \mu \mathrm{m})$ et non sphérique ; extrémité postérieure terminée par un dôme surmonté de 4 tubercules saillants.

Larve infestante longue $(310 \mu \mathrm{m})$, avec 4 languettes caudales égales.

Hôte : Miniopterus schreibersi, Nord Queensland, Australie.

\section{Genre Lukonema.}

En décrivant l'espèce type, L. lukoschusi Chabaud et Bain, 1974, nous avions émis l'hypothèse que la cellule osmo-excrétrice appendue à l'œsophage, provenait de la transformation de glandes œsophagiennes et nous pensions qu'il s'agissait de stichosomes latéro-ventraux.

Aucun matériel nouveau n'a été étudié, mais les données anatomiques acquises sur Muspicea et Riouxgolvania, en confirmant l'étroite filiation entre ces deux genres et Lukonema, nous conduisent à une interprétation un peu différente : l'appareil osmorégulateur apparaît bien formé à partir de glandes œsophagiennes, mais il s'agit de la glande dorsale. L'autre chaîne glandulaire appendue à l'œsophage (2 à 4 noyaux ?) correspond vraisemblablement aux glandes œsophagiennes latéro-ventrales fusionnées.

Chez la larve, le plan d'organisation est le même que chez Riouxgolvania et Muspicea (cf. fig. 2, A, in Chabaud et Bain, 1974) : glande dorsale volumineuse prolongée postérieurement par deux branches latérales ; juste en arrière de cette glande, deux petites cellules correspondent aux glandes œsophagiennes latéro-ventrales; à mi-corps, l'ébauche génitale, précédemment interprétée comme l'ébauche intestinale ; le trophosome lui-même, passé inaperçu sur les larves « in toto », est représenté sur la coupe $\mathrm{H}$ de la figure 2 ; la bouche est déportée ventralement et il existe, comme chez Riouxgolvania, une armature céphalique saillante.

IV. Genre Pennisia n. gen.

Le matériel a deux origines :

- un lot (Rom 645 889), parasite de la membrane alaire de Tadarida fulminans, pro- 


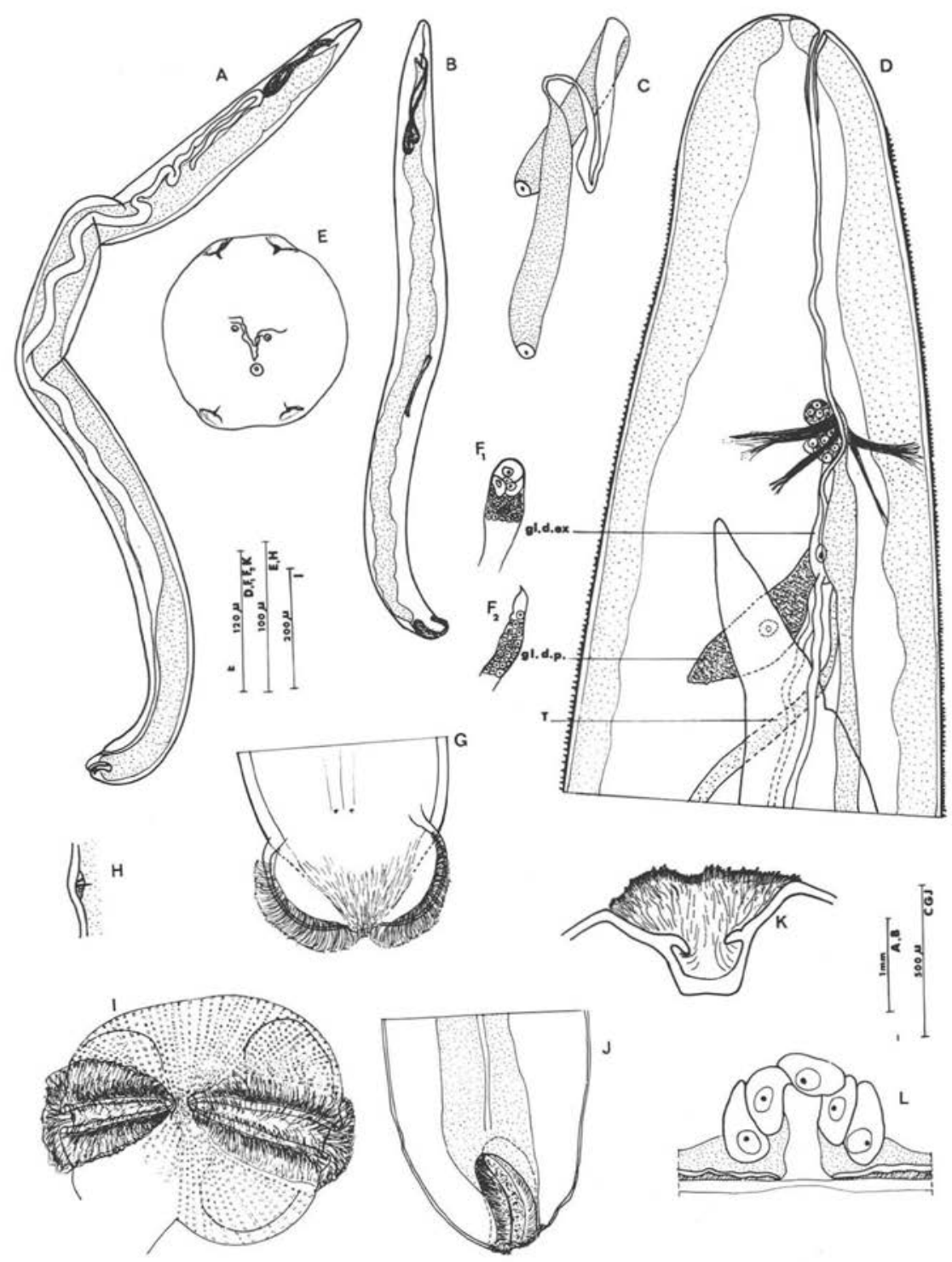

Fig. 6. Pennisia nagorseni $\mathrm{n}$. gen., n. sp. A : $\delta^{\star}$ allotype immature, vue latérale; B : très jeune $\delta$ à ébauche génitale médiane; $C$ : ébauche génitale d'une $q$ immature longue de $9 \mathrm{~mm}$;

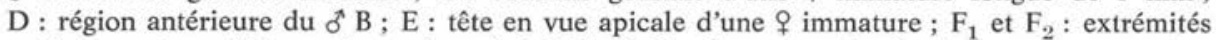
antérieure et postérieure du cordon génital du $\delta$ B ; G : queue du $\delta$ allotype, vue ventrale ; $\mathrm{H}: 1$ papille caudale du $\delta$ allotype, vue latérale; $\mathrm{I}$ : autre spécimen, queue avec formations plumeuses et phasmides, vue apicale; $\mathrm{J}$ : idem, vue latérale; $\mathrm{K}$ : coupe transversale optique d'une formation plumeuse; L: région vulvaire de la $q$ holotype, vue latérale. (A et B : éch. $1 \mathrm{~mm}$; C, G et J : éch. $500 \mu \mathrm{m}$; I : éch. $200 \mu \mathrm{m} ; \mathrm{D}, \mathrm{F}_{1}, \mathrm{~F}_{2}$ et $\mathrm{K}$ : éch. $120 \mu \mathrm{m}$; $\mathrm{E}, \mathrm{L}$ et $\mathrm{H}$ : éch. $100 \mu \mathrm{m})$ 
venant de Chipulo Cave en Rhodésie. Il comprend une femelle et un mâle immatures lot type 352 JE, déposé au Royal Ontario Museum, Toronto, Canada;

— un lot (Rom 71204), parasite de la membrane alaire de Tadarida africana, provenant de Nyanga Hill au Kenya. Il comprend un mâle et six femelles immatures éclatés ( $\left.\mathrm{n}^{\circ} 350 \mathrm{JE}\right)$ et un mâle immature monté entre lame et lamelle ( $\left.{ }^{\circ} 351 \mathrm{JE}\right)$.

Aucune différence morphologique n'ayant pu être mise en évidence entre les spécimens des deux lots, nous les plaçons dans la même espèce.

Chez les deux sexes, le corps est presque droit, légèrement aminci dans la région antérieure. La tête porte quatre papilles saillantes et deux petites amphides sub-apicales; la bouche est déplacée ventralement (fig. 6).

\section{Appareil digestif et appareil osmo-excréteur.}

L'œsophage est constitué par un simple canal cuticulaire qui se termine juste après l'anneau nerveux. Plusieurs glandes sont appendues à l'œsophage :

a) dorsalement, une glande uninucléée, traversée par un canal qui se prolonge tout le long du corps par deux canaux latéraux; la position et la structure de cette glande permettent de l'assimiler à la glande œsophagienne dorsale osmo-excrétrice. Celle-ci est suivie par une grande cellule au cytoplasme spongieux, la deuxième glande dorsale.

b) deux immenses glandes œsophagiennes unicellulaires, latéro-ventrales (580 à $1000 \mu \mathrm{m})$, qui pendent dans la cavité générale du ver.

La cavité générale du Nématode est occupée par un cordon épais et riche en réserves, le trophosome intestinal ; il n'y a pas d'anus.

\section{Appareil génital.}

a) Femelles : l'ébauche génitale est représentée par deux cordons cellulaires, aux extrémités identiques, reliés par un tube ; elle est médiane et longue de $680-750 \mu \mathrm{m}$ chez les vers de 6-7 mm et emplit tout le corps chez ceux de 13-14 mm ; chez ces grands spécimens, les ébauches de la vulve et de l'ovéjecteur sont formées ; elles sont situées à micorps.

b) Mâles: nous assimilons à des mâles immatures deux spécimens de $6,9 \mathrm{~mm}$ et $6,7 \mathrm{~mm}$ de long, qui ont une ébauche génitale médiane, longue de 680-750 $\mu \mathrm{m}$, caractérisée par des extrémités distales différentes : l'extrémité antérieure est arrondie avec quatre volumineuses cellules, l'extrémité postérieure est pointue avec une seule cellule (fig. $6, F_{1}$ et $F_{2}$ ). Chez le $\delta$ allotype long de $11,1 \mathrm{~mm}$, la glande génitale est formée par un unique et long cordon, dont l'extrémité distale est fermée. Aucun spicule ni autre organe copulateur n'est visible. Deux petites papilles sous-cuticulaires sont situées ventralement, à la hauteur de l'extrémité distale du tube génital

\section{Formations caudales.}

La queue est ornée par deux formations latérales d'aspect plumeux qui prennent naissance près de l'extrémité caudale, au niveau de deux petites cavités situées en profondeur sous la cuticule. 
Sur toute sa longueur, la " plume " est insérée le long de sa ligne médiane au fond d'une gouttière et elle est formée par un feuillet cuticulaire fin, dont les plis serrés ont l'aspect de villosités.

En raison de leur origine sous-cuticulaire et de leur position latérale, nous supposons que ces deux formations plumeuses dérivent des phasmides.

\section{Dimensions :}

- femelle holotype : corps long de $14,8 \mathrm{~mm}$ et large de $650 \mu \mathrm{m}$; anneau nerveux à $300 \mu \mathrm{m}$ de la tête; œsophage long de $300 \mu \mathrm{m}$; glandes latéro-ventrales longues de $570 \mu \mathrm{m}$; ébauche vulvaire à $7,3 \mathrm{~mm}$ de la tête ; formation plumeuse caudale longue de $350 \mu \mathrm{m}$;

— mâle allotype : corps long de $11,1 \mathrm{~mm}$ et large de $580 \mu \mathrm{m}$; anneau nerveux à $300 \mu \mathrm{m}$ de la tête ; œsophage long de $300 \mu \mathrm{m}$; glandes latéro-ventrales longues de $950 \mu \mathrm{m}$; papilles caudales à $425 \mu \mathrm{m}$ de l'extrémité caudale.

\section{Discussion.}

Ce Nématode, par sa localisation dans les ailes des Microchiroptères et par ses caractères anatomiques fondamentaux, se classe dans les Muspiceidae à proximité de Riouxgolvania et de Lukonema.

Les spécimens mâles et femelles dont nous disposons ont un orifice génital qui n'est pas perméable et une ébauche génitale qui reste très immature ; nous interprétons ces Nématodes comme des adultes immatures, mais peut-être s'agit-il en réalité de larves âgées.

Nous ne savons donc pas comment s'effectue la ponte; nous ignorons également si le mâle mûr acquiert une structure génitale plus élaborée que celle que nous connaissons. En dépit de ces incertitudes, ce Nématode nécessite la création d'un nouveau genre, du fait qu'il est dioïque et qu'il possède des formations caudales très originales.

Nous proposons donc la définition suivante : Pennisia $\mathrm{n}$. gen., Muspiceidae, à sexes séparés; 2 glandes œsophagiennes dorsales, dont l'antérieure est osmo-excrétrice, et deux glandes latéro-ventrales unicellulaires très développées; extrémité caudale portant une paire latérale de formations villeuses en forme de plume; espèce type : $P$. nagorseni $\mathrm{n}$. sp., parasite đe Microchiroptères éthiopiens.

\section{Conclusions générales}

Depuis le travail fondamental de Brumpt en 1930, nos connaissances sur l'ensemble de la famille des Muspiceides n'ont progressé que par l'étude de matériaux fragmentaires (1 $q$ immature de Riouxgolvania en 1968, des coupes histologiques de Lukonema en 1974), qu'il était difficile d'interpréter, car il s'agit d'animaux très atypiques. 
Disposant maintenant d'un matériel plus varié et plus abondant, il devient possible d'avoir une idée plus générale sur la famille et d'infirmer ou de confirmer un certain nombre des interprétations hypothétiques formulées précédemment.

\section{A. Morphologie comparée des 4 genres.}

1. Esophage et glandes œsophagiennes.

Notre nouvelle interprétation de cet appareil est représentée sur la figure 7.
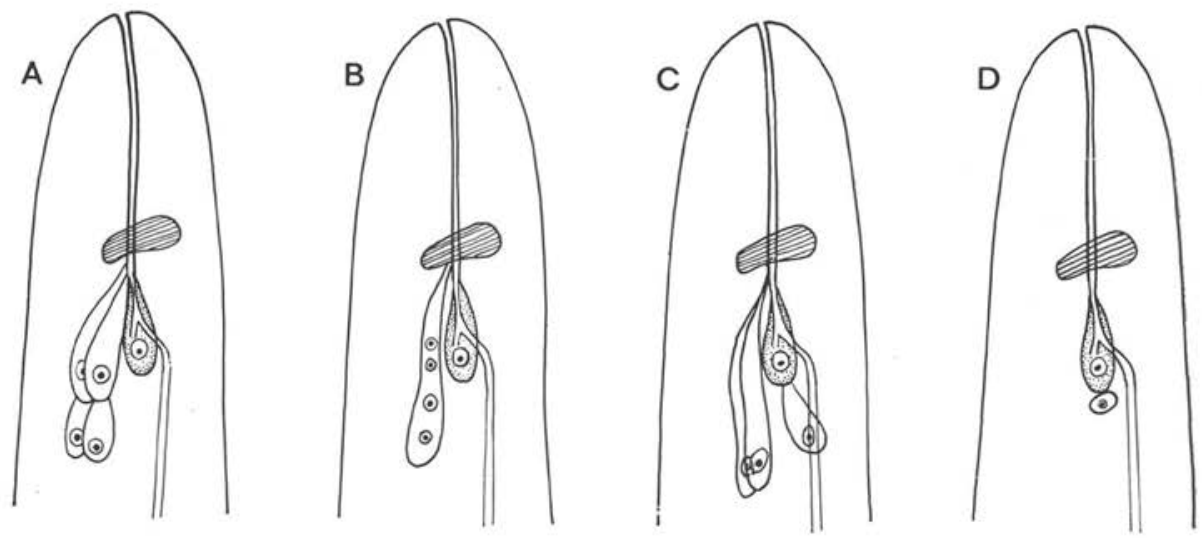

Fig. 7. Schéma de l'organisation de la région œsophagienne des 4 genres de Muspiceidae, vue latérale. A : Muspicea : B : Lukonema; C: Pennisia n.g.; D : Riouxgolvania.

De façon constante, la première glande œsophagienne dorsale est transformée en une cellule osmo-excrétrice qui reçoit les canaux excréteurs latéraux, longitudinaux, et qui se déverse dans la lumière œsophagienne. Il s'agit donc là d'un élément original et très caractéristique de la famille. A cette glande osmo-excrétrice peut être appendue une deuxième cellule glandulaire (Pennisia et Riouxgolvania).

Les glandes œsophagiennes latéro-ventrales sont plus variables : 2 paires de cellules chez Muspicea, 2 paires apparemment fusionnées chez Lukonema, 1 paire chez Pennisia, cellules atrophiées chez Riouxgolvania mûr.

\section{Trophosome.}

Dans les quatre genres, l'intestin est transformé en un trophosome, prenant naissance à proximité de l'extrémité postérieure de l'œsophage et se terminant dans la région caudale. Il n'y a pas d'anus.

3. Appareil génital.

Muspicea, Lukonema et Riouxgolvania sont protérandriques. La spermatogénèse paraît s'effectuer sur les parois du tube génital et est terminée au moment où les cellules distales de chaque cordon génital entrent en division pour effectuer l'ovogénèse. 
Les larves sont de grande taille, et mûrissent de façon relativement synchrone dans la poche utérine jusqu'au début du $3^{\text {e }}$ stade larvaire (ce fait n'a pu être vérifié que chez R. rhinolophi).

Lukonema et Riouxgolvania ont une vulve dans la région moyenne du corps, par où passent les larves, mais celles-ci, emprisonnées entre 2 assises cuticulaires, s'acheminent activement vers l'extrémité antérieure du Ver. Elles quittent la chauve-souris, hôte, en utilisant la perforation cutanée effectuée par la tête de la $q$ à la maturité.

Chez Muspicea, la vulve est atrophiée; les larves quittent directement le corps de la femelle par rupture de son extrémité antérieure, qui, ici aussi, a traversé l'épithélium cutané de la souris-hôte au moment de la maturité.

Pennisia est le seul genre dioïque; nous ne connaissons pas encore les formes mûres, mais la structure de la vulve des spécimens immatures et les grandes analogies avec Riouxgolvania et Lukonema laissent supposer un mécanisme de ponte analogue.

\section{Phasmides.}

Dans les 3 genres où les larves sont connues, celles-ci ont des phasmides comparables : 1 paire de grandes cellules latérales reliées chacune à un orifice plus postérieur.

Chez les adultes de Muspicea et Riouxgolvania, ces phasmides sont peu perceptibles; elles sont au contraire très remarquables chez Lukonema et Pennisia, et pourraient constituer des organes d'absorption.

\section{Larves.}

Dans les 3 genres où elles sont connues, les larves paraissent mûrir jusqu'au $3^{\mathrm{e}}$ stade larvaire dans l'utérus maternel; elles sont peu différentes d'un genre à l'autre et, à part l'appareil génital, offrent les mêmes traits d'organisation que l'adulte.

La seule différence importante réside dans la tête, qui est inerme chez Muspicea et armée d'un relief chitinoïde médian chez Riouxgolvania et Lukonema.

\section{B. Biologie.}

A partir de l'étude morphologique de Lukonema, nous avions supposé en 1974 que les cycles de Muspiceides étaient des cycles à un hôte. Les larves qui sortent de l'hôte par effraction cutanée n'auraient besoin que d'un court séjour à l'extérieur pour être infestantes ; l'infestation aurait lieu par voie cutanée chez Lukonema et par voie digestive (léchage) chez Muspicea.

Les données complémentaires obtenues depuis cette date nous permettent de rattacher les biologies de Riouxgolvania et, très vraisemblablement, de Pennisia, à celle de Lukonema. L'armature céphalique des larves des genres parasites de Chauves-souris, s'opposant à la bouche inerme de celles parasites de Rongeurs, semble venir à l'appui de ces hypothèses. Nous n'avons pas eu la possibilité de réaliser d'infestations expérimentales, mais nous avons pu vérifier cependant que, contrairement à Muspicea, les larves de $R$. rhinolophi sont très résistantes et ne présentent aucune altération après avoir passé 15 jours dans l'eau. 


\section{Affinités.}

Les 4 genres étudiés ci-dessus constituent un ensemble très homogène et très atypique pour des parasites de Vertébrés. Le seul parasite de Vertébré qui paraisse proche est Robertdollfusa paradoxa Chabaud et Campana, 1950, parasite d'Oiseaux, dont les structures sont trop atrophiées pour être utilisables dans la recherche des affinités.

L'habitus général de ces Nématodes, avec leur trophosome et leurs grandes larves libres dans l'utérus, évoquent plus des parasites d'Invertébrés, comme l'avait indiqué Brumpt dès 1930.

L'élément le plus caractéristique paraît être l'œsophage et ses glandes annexes. Il existe, chez presque tous les Dorylaimina parasites au sens de Chitwood (in Chitwood et Chitwood, 1950), 3 cellules particulières situées en avant des stichocytes proprement dits. La dorsale, parfois absente, semble en tout cas toujours nettement différente des 2 subventrales et la nomenclature de ces glandes est variée : "primary glands " (Chitwood et Chitwood, 1950), " pharyngeal glands» (Christie, 1936), « companion cells » (Hudson, 1971), ou " paired penetration glands » pour les glandes subventrales et «stichosome accessory structure" pour la glande dorsale (Poinar et Hess, 1974).

Il n'y a donc aucun doute sur l'appartenance des Muspiceidae aux Dorylaimina sensu Chitwood. Parmi ceux-ci, ce sont les Mermithoidea qui paraissent les plus proches, bien que la physiologie de chacune de ces glandes œsophagiennes antérieures paraît être très différente d'un groupe à l'autre.

\section{Bibliographie}

Bain O., Chabaud A.-G. (1968): Description de Riouxgolvania rhinolophi n.g., n.sp., Nématode parasite de Rhinolophe, montrant les affinités entre Muspiceoidea et Mermithoidea. Ann. Parasitol. Hum. Comp., 43, 45-50.

Brumpt E. (1930): Muspicea borreli Sambon, 1925, et cancers des souris. Ann. Parasitol. Hum. Comp., 8, 309-341.

Chabaud A.-G., Bain O. (1974) : Données nouvelles sur la biologie des Nématodes Muspiceides fournies par l'étude d'un parasite de Chiroptères: Lukonema lukoschusi n. gen., n. sp. Ann. Parasitol. Hum. Comp., 48, 819-834.

Chabaud A.-G., Campana Y. (1950): Nouveau parasite remarquable par l'atrophie de ses organes : Robertdollfusa paradoxa (Nematoda incertae sedis). Ann. Parasitol. Hum. Comp., 25, 325-334.

Chitwood B. G., Chitwood M. B. (1950): An introduction to Nematology, Monumental Printing Co., Baltimore, Md, $372 \mathrm{pp}$.

Christie J. R. (1936) : Life history of Agamermis decaudata, a Nematode parasite of grasshoppers and other insects. J. Agric. Research, Washington, 52, 161-198.

Hudson K. E. (1971): The form and function of the csophageal region of the insect parasite Tetradonema plicans (Nematoda: Tetradonematidae). Parasitology. 63, 137-143.

Mudry D. R., Dalley M. D. (1969) : Phlyctainophora squali sp.nov. (Nematoda, Philometridae) from the spiny dog-fish, Squalis acanthias. Proc. Helminth. Soc. Wash. 36, 280-284.

Poinar G. O., Hess R. (1974): Structure of the preparasitic juveniles of Filipjevimermis leipsandra and some other Mermithidae (Nematoda). Nematologica, 20, 163-173. 